
Application of the Montecarlo Simulation for the Implementation Analysis of
Fast Charging Stations for Electric Vehicles in the Galapagos Province

\title{
Aplicación de la Simulación de Montecarlo para el Análisis de la Implementación de Estaciones de Carga Rápida para Vehículos Eléctricos en la Provincia de Galápagos
}

\author{
L.A. Mera ${ }^{1} \quad$ P.E. Otero ${ }^{1} \quad$ V.E. Calle ${ }^{2}$ \\ ${ }^{1}$ Escuela Politécnica Nacional, Facultad de Ingeniería Eléctrica y Electrónica, Quito, Ecuador \\ E-mail: luis.mera01@epn.edu.ec; patricia.otero@epn.edu.ec \\ ${ }^{2}$ Agencia de Regulación y Control de Electricidad, Dirección Nacional de Estudios Eléctricos y Energéticos, Quito, \\ Ecuador \\ E-mail:emilio.calle@ekutron.com
}

Abstract

The present analysis starts with a review of applicable national and international standards for electric vehicles, in order to establish minimum requirements and technical parameters for the implementation of fast charging stations in the Galapagos Islands.

Based on the information of electrical energy production, vehicle demand and power demand in Galapagos, a Montecarlo simulation is carried out, considering parameters such as charging time probability, percentage of battery charge, and the charging curves of electric vehicles suitable for the islands, thus determining the required demand in two study cases.

In the first study case, the replacement of diesel buses by electric buses was considered; the second assumed the replacement of buses, SUVs and sedans.

The results of the demand analysis obtained from the Montecarlo simulation determined which study case has the mayor impact in the maximum demand curve and the number of vehicles that causes it, to later determine the number of stations and chargers required by each station, in addition of the location, considering the transit of the Galapagos Islands.

Index terms-- Montecarlo simulation, electric vehicle, charging station, charging curve, maximum demand, charging stations placement.
Resumen

El presente análisis parte de una revisión de normas nacionales e internacionales aplicables para vehículos eléctricos, con el fin de establecer requisitos mínimos y parámetros técnicos para la implementación de estaciones de carga rápida en las Islas Galápagos.

En base a información de generación de energía eléctrica, demanda vehicular y demanda de potencia en Galápagos, se realiza una simulación de Montecarlo, tomando en cuenta la probabilidad de hora de carga, porcentaje de carga en el que se encuentra la batería y curvas de carga de vehículos eléctricos aptos para las islas, así se determina la demanda requerida, considerando dos casos de estudio.

En el primero caso de estudio, se consideró el reemplazo de autobuses a diésel por autobuses eléctricos; el segundo, asumió el reemplazo de vehículos clase: autobús, SUV y automóvil.

Los resultados del análisis de demanda proveniente de la simulación determinaron qué caso de estudio tiene mayor impacto sobre la curva de demanda máxima y el número de vehículos que lo provoca, para posteriormente determinar el número de estaciones $y$ cargadores requeridos por cada estación, además de la ubicación de estas, considerando el tránsito de las islas Galápagos.

Palabras clave- Simulación de Montecarlo, vehículo eléctrico, estación de carga, curva de carga, demanda máxima, ubicación de estaciones de carga. 


\section{INTRODUCCIÓN}

Uno de los objetivos de desarrollo sostenible a nivel mundial es la acción frente al cambio, que propone limitar las emisiones de $\mathrm{CO} 2$ para prevenir que la temperatura global llegue a un aumento de $2{ }^{\circ} \mathrm{C}$ hasta el final del siglo [1]. En este contexto, es crucial el uso de electricidad proveniente de fuentes renovables, en lugar de combustibles fósiles, principalmente en el sector del transporte [2]. La industria automotriz alineada a esta premisa ha desarrollado en los últimos años vehículos eléctricos (VE) más eficientes y económicos, habilitando la posibilidad de hacer extensivo su uso [2]. Sin embargo, aún existen algunos limitantes, tales como el requerimiento infraestructura para recarga de baterías, ya sea carga lenta a nivel doméstico o carga rápida y ultrarápida a nivel comercial, electrolineras de se requiere infraestructura de carga rápida para las baterías, dado que el tiempo de abastecimiento es un factor crítico que se interpone en el camino para la aceptación generalizada de los VE [3].

Por su parte, el gobierno del Ecuador a través de la ley Orgánica de Eficiencia Energética, ha establecido que a partir del 2025 el servicio de transporte público urbano e interparroquial será eléctrico. Para las islas Galápagos se ha impulsado la iniciativa "Cero Combustibles Fósiles", dirigida a reducir las emisiones de $\mathrm{CO} 2$ a través de la implementación de generación eléctrica con fuentes renovables como la solar y eólica. Estas dos políticas posibilitarían la inserción de un parque automotriz totalmente eléctrico y el aprovechamiento de fuentes renovables en el archipiélago de las Islas Galápagos, que se caracteriza por ser una zona fauna y flora sensibles a la interferencia humana, estar aisladas del continente y tener una limitada cantidad de recursos energéticos.

La futura demanda de energía de lo VE y la infraestructura requerida hace imprescindible realizar un análisis técnico de la normativa aplicable al caso de las islas y considerar la optimización de la demanda en la infraestructura de carga de los VE para evitar sobredimensionar la expansión del sistema eléctrico.

\section{NORMATIVA}

Como base para la implementación de infraestructura de estaciones de carga para VE, se debe utilizar una normativa que permita homologar los componentes principales, tanto de los VE, como de la infraestructura. La normativa debe ser aplicable a las condiciones del caso Galápagos, por los que se procede a revisar los criterios establecidos en la normativa nacional e internacional.

Un primer criterio se toma de la Comisión Internacional Electrotécnica, IEC por sus siglas del inglés (International Electrotechnical Commission), que presenta los segmentos para homologación de la Fig. 1.

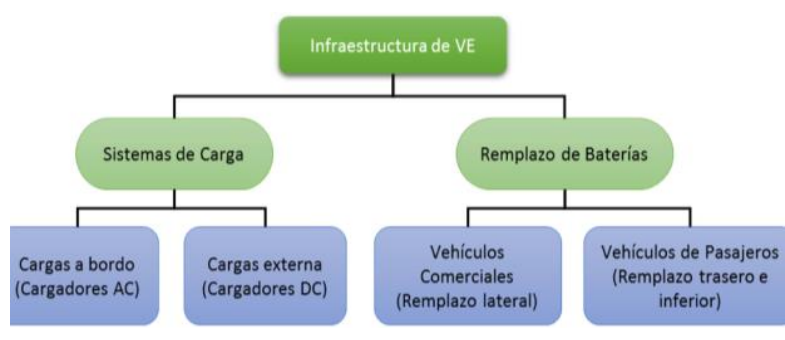

Figura 1: Segmentos de infraestructura a homologar

Por otra parte, a nivel mundial se consideran cuatro criterios fundamentales para normalizar una infraestructura de carga destinada a VE: seguridad, comunicación, compatibilidad y rendimiento. Bajo estos criterios, se analiza lo establecido en las siguientes normas internacionales y nacionales:

- IEC 61851-1: Esta norma se centra en requisitos generales para el sistema de carga conductiva de vehículos eléctricos. Entre los aspectos más importantes que se puede encontrar está modo de carga, tipos de carga, conectividad a la red, etc. [4].

- ISO 17409: Norma dedicada a requisitos de seguridad para vehículos que se conectan a una red externa de alimentación [5].

- IEC 61851-22: Estación de carga de vehículos eléctricos dedica exclusivamente para conexión AC, resaltan las funciones de seguridad que las estaciones de seguridad AC deben poseer [6] [7].

- IEC 61851-23: Estación de carga de vehículos eléctricos dedica exclusivamente para conexión DC, resaltan las funciones de seguridad que las estaciones de seguridad DC deben poseer [7].

- ISO 6469-3: Protección de personas contra riesgos eléctricos. Especificaciones de seguridad [8].

- Código Eléctrico Nacional (NEC): En este se describe aspectos generales de las estaciones de carga, además de presentar aspectos para las instalaciones eléctricas de las mismas refiriéndose en mayor parte a instalaciones residenciales [9].

- Norma Ecuatoriana de Construcción: En esta norma se presenta aspectos que rigen en el país referente a instalaciones eléctricas en medio y bajo voltaje [10].

- IEEE Estándar 80-2000: Estándar que contiene requisitos básicos y guía de diseño para sistemas de puesta a tierra [11].

- IEC 60364-7-722: Instalaciones eléctricas de bajo voltaje; requisitos para instalaciones o ubicaciones especiales [12].

- Comité Europeo de Normalización Electrotécnica (CENELEC): Presenta aspectos como tipos de conectores, modos de carga, tipos de carga, potencia de suministro, tipos de cargadores, entre otros [13].

- Guía Técnica-Estrategia de transporte sostenible USA: Consideraciones y aspectos para la operación e instalaciones de estaciones de carga para vehículos eléctricos [14].

- IEC 62840-2: Proporciona requisitos de seguridad para los sistemas de intercambio de batería en VE 
con fuente de alimentación de hasta $1000 \mathrm{~V}$ en corriente alterna o de hasta $1500 \mathrm{~V}$ en corriente continua. La norma es aplicable a sistemas de intercambio de baterías para sistemas de almacenamiento en el sitio [15].

- IEC 60529: Grados de protección para armarios y tableros (Código IP) [16].

De la IEC 61851-1 se extrae también los posibles modos de carga [4]:

- Modo 1. Método de carga de AC que se realiza a través de una toma de corriente domestica con un enchufe estándar, no es de uso exclusivo para cada carga de vehículos eléctricos.

- Modo 2. Es un método de carga de AC, se diferencia del Modo 1 por la incorporación de un sistema de protección y un interruptor diferencial en el cable. Este modo incorpora un control de carga en caso de existir una mala conexión entre el VE y la red eléctrica.

- Modo 3. Método de carga de AC, se conecta directamente a la red eléctrica a través de un circuito dedicado. Sus características son: alto grado de comunicación entre el VE y la red eléctrica, mayor seguridad.

- Modo 4. Método de carga de DC, el cual utiliza un cargador externo que permite una velocidad de carga rápida. En el punto de carga se utiliza un conversor de AC/DC, este método se utiliza en las estaciones de carga debido a que se maneja potencias superiores a los $50 \mathrm{~kW}$. Posee un sistema de protección y control de carga.

\subsection{Parámetros de las estaciones de carga}

Con base a los alcances de la normativa antes referidas, se considera que la infraestructura de las estaciones de carga rápida a implementarse en las islas Galápagos debe tener los siguientes elementos:

1. Contar con un mínimo de dos estaciones para cargar de manera simultánea un VE categoría $\mathrm{M}^{1}$ y un vehículo eléctrico de categoría $\mathrm{N}^{2}$.

2. El método de carga que se debe emplear debe ser el conductivo, es decir con una conexión física entre el VE y la estación de carga.

3. La conexión entre el VE y la red de suministro de energía eléctrica debe emplear un cable y un conector que estén fijos en la estación de carga y cuyo propósito sea la conexión en la entrada del VE y posterior carga de la batería.

4. El cargador a emplearse en las estaciones de las islas Galápagos, se ha seleccionado tomando en cuenta los VE que admiten carga rápida y están disponibles

${ }^{1}$ Categoría M: Vehículo automotor que posee al menos 4 ruedas utilizado para el transporte de pasajeros. comercialmente [17]. La Tabla 1 presenta un resumen los VE existentes en las islas en el 2018, e indica que el $77 \%$ de vehículos emplea un cargador SAE combo 2 de base combinada (CCS), $17 \%$ emplea un cargador CHAdeMO (CDM) y $6 \%$ emplea supercargadores Tesla (TS); por tanto, el cargador que debe empelarse en las estaciones de carga rápida debe ser el SAE combo 2 de base combinada, Fig. 2.

5. Los cargadores deberán tener una potencia igual o mayor a $22 \mathrm{~kW}$, sean estos en corriente alterna o corriente continua.

Tabla 1: Parque automotor de las principales islas en el 2018 [17]

\begin{tabular}{|c|c|c|c|c|}
\hline \multicolumn{5}{|c|}{ Vehículos con disponibilidad de carga rápida } \\
\hline Marca & Vehículo & Origen & Año & Cargador \\
\hline \multirow{2}{*}{ Audi } & e-tron 50 & Europa & 2020 & $\mathrm{CCS}$ \\
\hline & e-tron 55 & Europa & 2019 & $\mathrm{CCS}$ \\
\hline \multirow{2}{*}{ BMW } & $\mathrm{i} 3$ & Europa & 2018 & CCS \\
\hline & $\mathrm{i} 3 \mathrm{~s}$ & Europa & 2018 & CCS \\
\hline Citroen & C-zero & Europa & 2016 & CDM \\
\hline DS & 3 Crossback e-tense & Europa & 2020 & CCS \\
\hline \multirow{2}{*}{ Ford } & Focus Electric & América & 2017 & CCS \\
\hline & Mustang Mach-E & América & 2020 & CCS \\
\hline $\begin{array}{c}\text { Harley- } \\
\text { Davinson }\end{array}$ & Livewire & América & 2020 & CCS \\
\hline Honda & $\mathrm{e}$ & Asia & 2020 & CCS \\
\hline \multirow{2}{*}{ Hyundai } & Ioniq Electric & Asia & 2016 & CCS \\
\hline & Kona Electric & Asia & 2018 & CCS \\
\hline Jaguar & I-pace & Europa & 2018 & $\mathrm{CCS}$ \\
\hline \multirow{2}{*}{ Kia } & e-Niro & Asia & 2019 & $\mathrm{CCS}$ \\
\hline & e-soul & Asia & 2019 & CCS \\
\hline $\begin{array}{c}\text { Mercedes } \\
\text { Benz }\end{array}$ & EQC & Europa & 2019 & $\mathrm{CCS}$ \\
\hline Mini & Eléctrico & Europa & 2020 & $\mathrm{CCS}$ \\
\hline Mitsubishi & Outlander & Asia & 2018 & CDM \\
\hline \multirow{2}{*}{ Nissan } & e-NV200 & Asia & 2018 & $\mathrm{CDM}$ \\
\hline & Leaf & Asia & 2018 & $\mathrm{CDM}$ \\
\hline \multirow{4}{*}{ Peugeot } & e-2008 & Europa & 2020 & $\mathrm{CCS}$ \\
\hline & e-208 & Europa & 2020 & $\mathrm{CCS}$ \\
\hline & iOn & Europa & 2016 & $\mathrm{CDM}$ \\
\hline & Partner Electric & Europa & 2017 & CDM \\
\hline Polestar & 2 & Europa & 2020 & $\mathrm{CCS}$ \\
\hline Porsche & Taycan & Europa & 2020 & CCS \\
\hline Renault & Zoe R110 ZEZ50 & Europa & 2020 & CCS \\
\hline Skoda & Citigoe iV & Europa & 2020 & CCS \\
\hline \multirow{3}{*}{ Tesla } & Model 3 & América & 2019 & CCS \\
\hline & Model S & América & 2019 & TS \\
\hline & Model X & América & 2019 & TS \\
\hline \multirow{3}{*}{ Volkswagen } & e-golf & Europa & 2017 & CCS \\
\hline & e-Up & Europa & 2016 & CCS \\
\hline & ID. 3 & Europa & 2020 & CCS \\
\hline Volvo & $\mathrm{XC} 40$ & Europa & 2020 & CCS \\
\hline
\end{tabular}

${ }^{2}$ Categoría N: Vehículo automotor que posee al menos 4 ruedas utilizado para transporte de carga. 


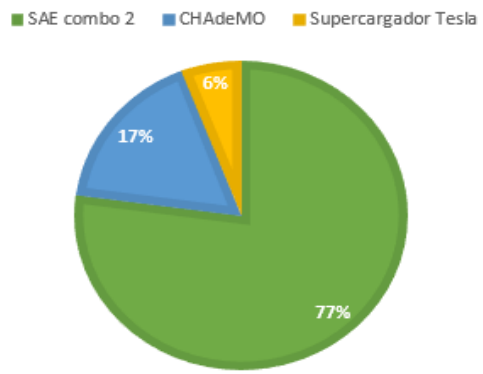

Figura 2: Cargadores empleados para carga rápida

6. El modo de carga hace referencia a la seguridad al momento de la carga y la gestión de potencia para cuidar la batería. Se escoge el Modo 3 o Modo 4 para estaciones de carga rápida $\mathrm{AC}$ y modo 4 para estaciones de carga rápida DC.

7. La operación de los modos de carga debe incluir funciones de seguridad obligatorias como:

- Detección de presencia de tierra

- Verificación de continuidad de la tierra

- Protecciones contra sobrevoltaje, bajo voltaje, sobrecorriente, cortocircuito y corriente de fuga

- Presencia de conector y bloqueo.

- Energización de la fuente de alimentación al VE

- Desenergización de la fuente de alimentación al VE

8. Se debe proporcionar un dispositivo de protección contra sobrecorriente (por ejemplo, fusible, disyuntor) en el circuito de alimentación del vehículo, este criterio se aplica para estaciones de carga AC y DC.

9. Se debe proporcionar un dispositivo de protección contra sobrecorriente (por ejemplo, fusible, disyuntor) dentro del cargador, este criterio se aplica para estaciones de carga AC y DC

10. Las cámaras de transformación que abastezcan a las estaciones para la movilidad eléctrica deben ser subterráneas.

11. El sistema de puesta a tierra de las debe estar diseñado acuerde al estándar IEEE 80-2000; para este caso debe tener una resistencia de puesta a tierra de un valor mínimo de $5 \Omega$.

\section{DIMENSIONAMIENTO DE ESTACIONES DE CARGA RÁPIDA}

\subsection{Simulación de Montecarlo}

La simulación de Monte Carlo es una técnica matemática computarizada para generar datos de muestra aleatorios basados en alguna distribución conocida para experimentos numéricos [18].

Este método puede usarse en aquellas situaciones en las que se necesita hacer una estimación y tomar decisiones sobre variables inciertas, como las predicciones del pronóstico del tiempo.
Características importantes:

- La salida debe generar muestras aleatorias.

- La distribución de entrada debe ser conocida.

- $\mathrm{Su}$ resultado debe ser conocido al realizar un experimento.

Ventajas:

- Proporciona un muestreo estadístico para experimentos numéricos usando la computadora.

- Proporciona una solución aproximada a problemas matemáticos.

- Se puede usar para problemas estocásticos y deterministas.

Desventaja:

- Consume mucho tiempo al ser necesario generar un gran número de muestras para obtener la salida deseada, y los resultados son solo la aproximación de los valores verdaderos, no los exactos [18].

\subsection{Teoría de colas}

Es el estudio matemático de la congestión y los retrasos de la espera en línea. La teoría de colas examina cada componente de la espera en línea para ser atendido, incluido el proceso de llegada, el proceso de servicio, la cantidad de servidores, la cantidad de lugares del sistema y la cantidad de clientes, que pueden ser personas, paquetes de datos, automóviles, etc [19].

\subsubsection{Proceso de llegada}

El proceso de llegada más simple es aquel en el que se tiene llegadas completamente regulares, es decir, el mismo intervalo de tiempo constante entre llegadas sucesivas. Un flujo de llegadas de Poisson corresponde a llegadas al azar. Hace referencia a:

- Cómo llegan los clientes, por ejemplo, solos o en grupos.

- Cómo las llegadas se distribuyen en el tiempo.

- Sí hay una población finita de clientes o un número infinito.

\subsubsection{Mecanismo de servicio}

Se refiere a una descripción de los recursos necesarios para que: el servicio comience, cuánto tiempo llevará el servicio, la cantidad de servidores disponibles,

Si se permite la preferencia, es decir, un servidor puede detener el procesamiento de un cliente para tratar con otro cliente de "emergencia".

Asumir que los tiempos de servicio para los clientes son independientes y no dependen del proceso de llegada es común. Otro supuesto común sobre los tiempos de servicio es que están distribuidos exponencialmente.

\subsubsection{Características de la cola}

- Balking: clientes que deciden no unirse a la cola si es demasiado larga.

- Renegar: los clientes dejan la cola si han esperado 
demasiado tiempo para recibir servicio.

- Compilación: los clientes cambian entre colas si piensan que recibirán servicio más rápido al hacerlo. Una cola de capacidad finita o de capacidad infinita [19].

\subsection{Procedimiento propuesto}

El dimensionamiento de las estaciones de carga rápida se ha realizado en base a la demanda máxima diversificada, con el objetivo de considerar la simultaneidad de los picos de carga de los diferentes vehículos eléctricos que se pretende introducir a la provincia de Galápagos. La metodología que se aplicó sigue los siguientes pasos:

1. Determinar las curvas de carga de los VE existentes, probados y cargados en Galápagos, en base a mediciones de potencia realizadas en el momento de la carga.

2. Determinar la clase, la probabilidad de ocurrencia y el número de VE que ingresarán a las islas en el periodo 2019-2028, teniendo en cuenta el parque automotor actual y el crecimiento de turistas y población, para las diferentes clases.

3. Determinar la probabilidad de cargar un VE en las diferentes horas del día, para este punto se ha tomado en cuenta el costo de la energía eléctrica establecido en el pliego tarifario y las horas de aprovechamiento de generación fotovoltaica. El aprovechamiento solar se considera dada la proyección de la instalación de nuevos proyectos fotovoltaicos.

4. De la información de los pasos anteriores se realiza una simulación de Montecarlo con el objetivo de obtener escenarios de carga y determinar la demanda máxima diversificada para los casos de estudio.

5. De los casos de estudio, se selecciona la demanda máxima diversificada que causa la mayor influencia y el número de vehículos que la provocan.

6. En base al número de vehículos resultantes del paso 5 se ha dimensionado la estación o estaciones de carga que se instalaran en Galápagos. Este punto se realizó en base a la teoría de colas.

7. Se determina los lugares donde se ubicarán las estaciones de carga rápida para VE.

\subsection{Escenarios de estudio}

Se plantea dos escenarios de incorporación de VE, que toman lo indicado en la Ley de Eficiencia Energética, que dicta que hasta el 2025 todos los vehículos destinados al transporte masivo de pasajeros deben ser eléctricos. Dada la fecha del presente trabajo, el plazo da como resultado un periodo de 5 años.

Con esta premisa el primer escenario que se plantea es el remplazo paulatino de la flota de 56 buses concentrados en la isla Santa Cruz, teniendo en cuenta que estos se reemplazaran en su totalidad en el periodo 2020-2025, además se incluirá el aumento del parque automotor de autobuses en este mismo periodo y posterior proyección hasta el año 2028. Para la proyectar el crecimiento del parque automotor de autobuses se utilizará la tasa de crecimiento anual de turistas. Se consideran únicamente autobuses eléctricos de $12 \mathrm{~m}$.

El segundo escenario es el reemplazo paulatino de la flota de automóviles, SUV y autobuses pertenecientes a las islas de Santa Cruz y San Cristóbal, en este caso se tomará el periodo 2019-2025, dado que, a la fecha de este estudio, el reemplazo de los vehículos a gasolina por eléctricos ya ha empezado, posteriormente se proyectará el crecimiento del parque automotor hasta el 2028. Para proyectar el crecimiento de automóviles se utilizará la tasa de crecimiento anual de la población.

En ambos casos el objetivo será determinar la demanda máxima coincidente de grupo para posteriormente dimensionar la estación o estaciones de carga para la provincia de Galápagos, además se analizará el efecto de la inserción de vehículos eléctricos sobre la curva de carga la Empresa Eléctrica Galápagos.

\subsection{Modelo de simulación}

Basado en la simulación de Monte Carlo, el modelo que se presenta tiene como objetivo establecer curvas de carga en los escenarios previamente planteados, teniendo como base de información las curvas de carga de los VE obtenidas mediante mediciones y el número de vehículos de cada escenario. El algoritmo desarrollado realiza las siguientes etapas:

- Se ingresa el número de VE establecidos en cada escenario.

- Se utiliza la simulación de Montecarlo para generar valores de hora inicial de carga, porcentaje inicial de la batería del vehículo eléctrico y tipo de vehículo.

- Se asigna curvas de carga en función de la hora inicial de carga, porcentaje inicial de la batería del vehículo eléctrico y tipo de vehículo.

- Se realiza interacciones para obtener las curvas de carga.

Cada uno de los casos, el análisis se obtuvo con base al promedio de los resultados de 100 iteraciones. El algoritmo realiza las siguientes etapas:

- Utiliza la simulación de Montecarlo para generación de la hora inicial de carga, porcentaje inicial de batería y tipo de vehículo.

- Realiza la asignación de curvas de carga en función de la hora inicial de carga y porcentaje inicial de batería y tipo de vehículo.

- Ejecución de la cantidad de iteraciones deseadas para obtención de la curva de carga.

\subsubsection{Tipo de vehículo, energía y potencia de carga}

De la información de los VE existentes en Galápagos y del análisis de la sección 2.1, se considera tres tipos de vehículos cuyas potencias máximas de carga son: 1,99; 2,03 y $6,22 \mathrm{~kW}$; y para un autobús eléctrico de $12 \mathrm{~m}$ se toma una potencia máxima de carga de $80 \mathrm{~kW}$. En lo que 
corresponde a los tiempos de carga se elaboró una curva para análisis, que considera el comportamiento de cargadores que emplean el "Modo 3" y se referencia a las curvas obtenidas en estudios previos de la Agencia de Regulación y Control de Electricidad (ARCONEL) [20]. Estas curvas se presentan en la Fig. 3.

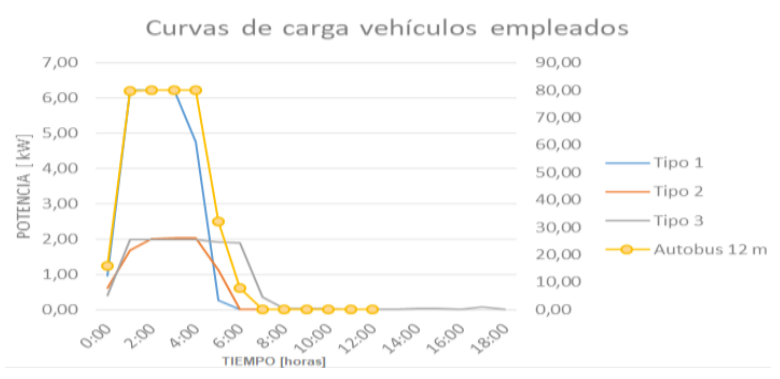

Figura 3: Curvas de carga, tiempo y potencia empleados

\subsubsection{Hora de carga}

La hora de carga de los vehículos eléctricos se realiza en torno a dos aspectos; el primero en base a la tarifa energética establecida por la ARCONEL y el segundo aspecto tiene como consigna aprovechar la generación fotovoltaica existente en Galápagos, especialmente en las islas San Cristóbal y Santa Cruz.

De los datos históricos disponibles al 2012 se evidencia que entre las 11:00 h y 13:00 h existe la máxima radiación solar en las islas Galápagos.

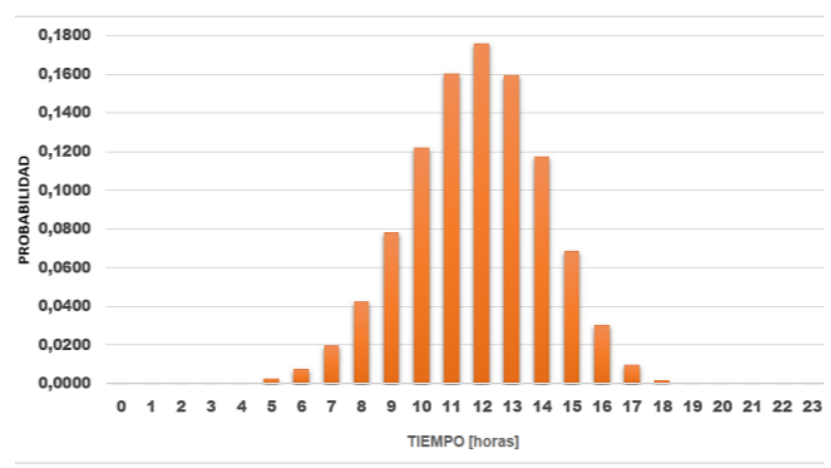

Figura 4: Distribución de probabilidad.

La determinación de la hora de carga corresponde a la distribución de probabilidad binomial de la Fig. 4 que tiene la siguiente expresión:

$$
P_{(x)}=\sum_{x=0}^{23} \frac{n !}{x !(n-x) !} p^{x} q^{n-x}=1
$$

donde $\mathrm{n}$ son las horas del día, $\mathrm{x}$ la hora carga, $\mathrm{p}$ la probabilidad de carga y q la probabilidad de no carga

\subsubsection{Intervalo de carga}

Como criterio de análisis se estima el comportamiento de los usuarios de VE, quienes acudirán a recargar antes de llegar a un $20 \%$ de carga y no asistirán si la carga es superior al $90 \%$, es decir la carga de vehículos se realizará cuando el sistema de carga se encuentre en el intervalo del $20 \%$ al $90 \%$, como se representa en la Fig. 5.

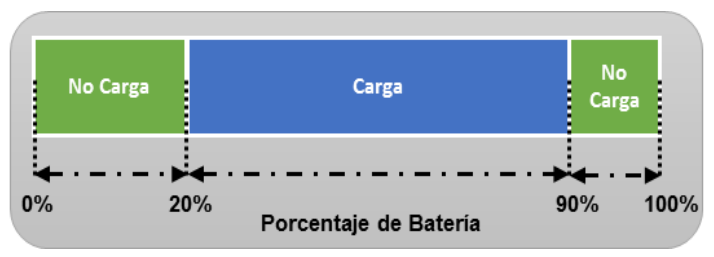

Figura 5: Intervalo de carga

\subsubsection{Cantidad de vehículos}

La cantidad de vehículos para los casos de estudio, previamente descritos, se los proyecta teniendo en cuenta lo siguiente:

- Para vehículos clase automóvil y SUV el crecimiento del parque automotor se correlaciona con el crecimiento anual de la población de las islas Galápagos, de esta manera el crecimiento estimado es de $2,53 \%$.

- Para vehículos clase autobús el crecimiento del parque automotor se correlaciona con el ingreso anual de turistas a Galápagos, de esta manera el crecimiento estimado es de 3,09\% [21].

En el 2018 el parque automotor de las clases de vehículos involucrados en las principales islas de Galápagos se muestra en la Tabla 2:

Tabla 2: Parque automotor 2018 en las principales islas [22]

\begin{tabular}{|c|c|c|c|c|c|}
\hline Isla & Total & $\begin{array}{c}\text { SUV a } \\
\text { gasolina }\end{array}$ & $\begin{array}{c}\text { Automóviles } \\
\text { a gasolina }\end{array}$ & $\begin{array}{c}\text { Automóviles } \\
\text { eléctricos }\end{array}$ & eSUV \\
\hline $\begin{array}{c}\text { Santa } \\
\text { Cruz }\end{array}$ & 484 & 203 & 138 & 116 & 27 \\
\hline $\begin{array}{c}\text { San } \\
\text { Cristóbal }\end{array}$ & 54 & 23 & 15 & 13 & 3 \\
\hline Isabela & 4 & 2 & 1 & 1 & 0 \\
\hline
\end{tabular}

El parque automotor de autobuses de Galápagos se concentra en la isla Santa Cruz con un total de 56 unidades [22].

\subsubsection{Evaluación de impacto}

La evaluación de impacto se realizó tomando en cuenta la demanda máxima total registrada por la empresa eléctrica Galápagos, dicha curva se obtiene tomando en cuenta el promedio de consumo de los 31 días de marzo de 2018, la cual se registró a las 19:00 con valores de 2.962,86 kW para el sistema de San Cristóbal y de $5.867,05 \mathrm{~kW}$ para Santa Cruz; a esta curva se agregan las obtenidas resultado de la incorporación de vehículos eléctricos según cada caso de estudio propuesto. Se usará la curva antes mencionada debido a la ausencia de datos en años previos al 2018 y por la poca colaboración de la empresa distribuidora al ofrecer lo mismos, lo cual no permitió hacer una proyección de la demanda de las islas mencionadas. Los perfiles de carga de estos sistemas se presentan en la Figura [20]. 


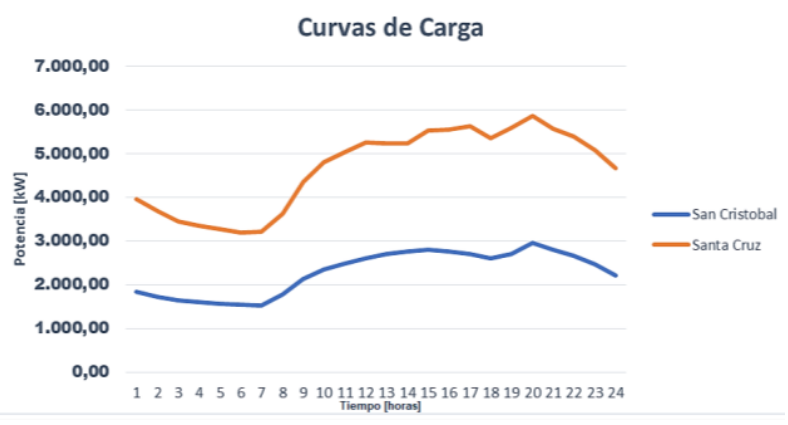

Figura 6: Perfiles de demandas máximas registradas

\subsection{Teoría de colas aplicación}

La estación de carga rápida para vehículos eléctricos es un sistema de servicio estocástico, siendo los cargadores los que brindan el servicio, el modelo del sistema de colas se muestra en la Figura. 7.

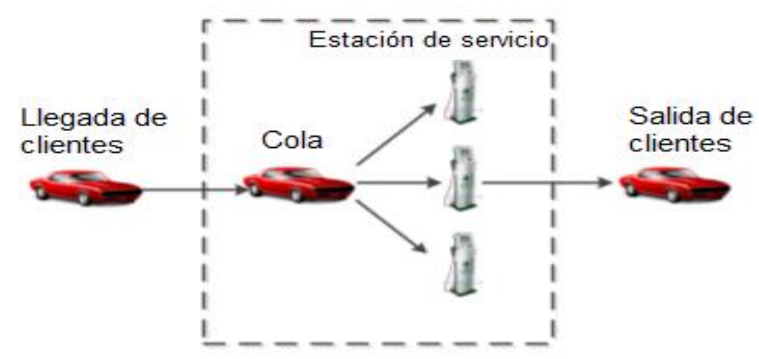

Figura 7: Esquema teoría de colas

- Sistema de entrada: la llegada de los VE a la estación de carga es un evento independiente y satisface condiciones de estacionalidad, propiedad de Márkov, y universalidad, por lo tanto, la llegada de VE a la estación se da de acuerdo con el proceso de Poisson y los intervalos de tiempo entre vehículos supone una distribución exponencial negativa.

- Sistema de salida: El tiempo de carga para cada VE depende de la energía restante en la batería, se utiliza una distribución exponencial negativa para describir el tiempo de carga.

- Disciplina de servicio: un cargador solo puede servir a un solo vehículo a la vez.

- Modelo de cola: la estación de carga es una cola estándar M/M/s, es decir se atiende por orden de llegada.

- Estación de servicio: puede servir a más de un vehículo a la vez al mismo tiempo.

- Tasa promedio de llegada: número de vehículos (n) que llegan a la estación por unidad de tiempo.

$$
\lambda=\frac{n}{\text { tiempo de apertura de la estación }}
$$

- Tasa de servicio promedio: número de clientes servidos por unidad de tiempo.

$$
\mu=\frac{1}{\text { tiempo promedio de carga }}
$$

El sistema se ve afectado por el estado inicial y por el tiempo que ha transcurrido desde el inicio, dándose una condición transitoria. Al transcurrir el tiempo suficiente el estado del sistema se vuelve independiente del estado inicial y del tiempo transcurrido volviéndose un estado estable y la distribución del sistema permanece igual con el tiempo. Por lo tanto, para cualquier $n$ la entrada debe ser igual a la salida. La probabilidad que satisface esta condición de acuerdo con [24] es:

$$
P_{o}=\left[\left(\sum_{i=0}^{s-1} \frac{\rho^{i}}{i !}\right)+\frac{\rho^{s}}{s !\left(1-\rho_{s}\right)}\right]^{-1}
$$

donde:

$$
\begin{gathered}
\rho=\frac{\lambda}{\mu} \\
\rho_{s}=\frac{\lambda}{\mu * s} \leq 1
\end{gathered}
$$

siendo s el número total de cargadores, que inicia en 0.

Los factores que inciden en la decisión del número de cargadores, los cuales se muestran a continuación:

a) El tiempo de estadía promedio a largo plazo por cliente.

$$
W_{s}=\frac{\rho^{s+1}}{\lambda(s-1)(s-\rho)^{2}} P_{o}+\frac{1}{\mu}
$$

b) El porcentaje de inactividad del cargador.

$$
I \%=1-\rho_{s}
$$

Existen dos formas de determinar el número óptimo de cargadores:

Método gráfico: el cual consiste en realizar curvas de Ws y Po con relación al número de cargadores s, con el objetivo de visualizar la variación de las mismas, el número óptimo de cargadores se escoge cuando las curvas ya no representan un cambio significativo (Fig. 8).

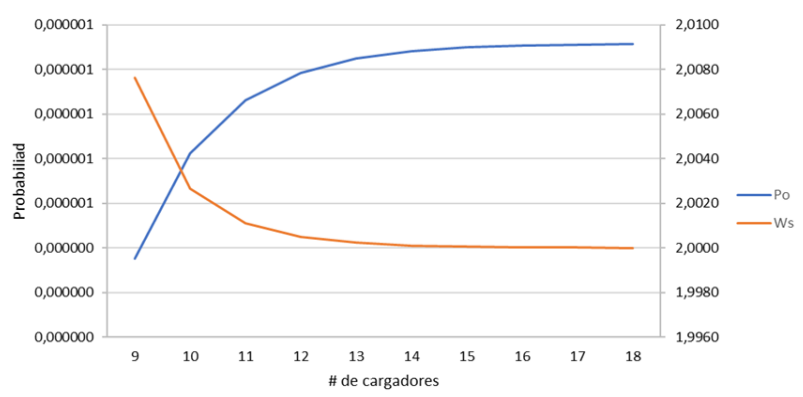

Figura 8: Método grafico para selección de numero de cargadores.

El segundo método consiste en tomar los índices sistemas más conflictivos, Ws y I\%, para determinar la cantidad óptima de servidores. Se debe satisfacer las siguientes condiciones:

$$
\begin{aligned}
& W_{s} \leq \propto \\
& I \% \leq \boldsymbol{\beta}
\end{aligned}
$$


Donde $\propto$ y $\boldsymbol{\beta}$ son factores que dependen del comportamiento de las personas al momento de esperar por lo que lo $\propto$ y $\boldsymbol{\beta}$ toman valores de 0,6 y $50 \%$ respectivamente [6].

\section{RESULTADOS}

\subsection{Potencia requerida}

\subsubsection{Caso 1}

Para el 2025 se ha reemplazo todo el parque automotor de autobuses que utilizan combustibles fósiles, además se considera el aumento del parque automotor, para el año 2028 se proyecta tener un total de 74 autobuses eléctricos de 12 metros.

En la Figura 9 se muestra el impacto de ingresar 74 autobuses eléctricos cuya demanda máxima diversificada es 2,49 MW, se ha incrementado $42,44 \%$ de la demanda máxima presentada en marzo de 2018, la demanda crece a partir de las 9:00 y empieza a decaer alrededor de las 15:00 teniendo el pico máximo entre las 14:00 y 15:00 horas.

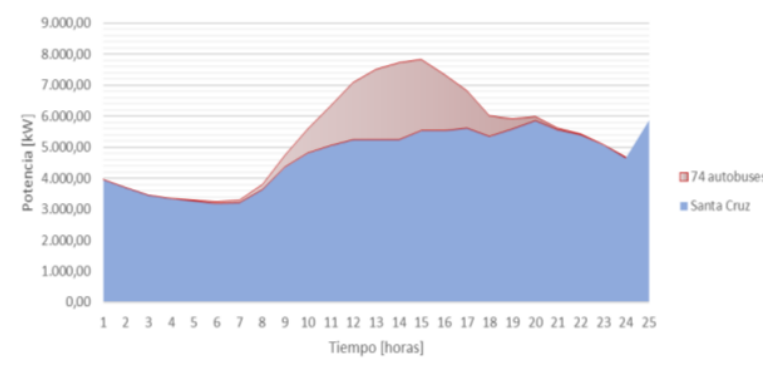

Figura 9: Demanda de potencia 74 autobuses eléctricos

\subsubsection{Caso 2}

Para el año 2028 se proyectó 712 vehículos eléctricos con lo que se tiene se tiene una demanda máxima diversificada de 3,318 MW para el caso de Santa Cruz; mientras que para el caso de San Cristóbal se tiene para el año 2028 con 74 vehículos eléctricos se tiene se tiene una demanda máxima diversificada de $100,74 \mathrm{~kW}$.

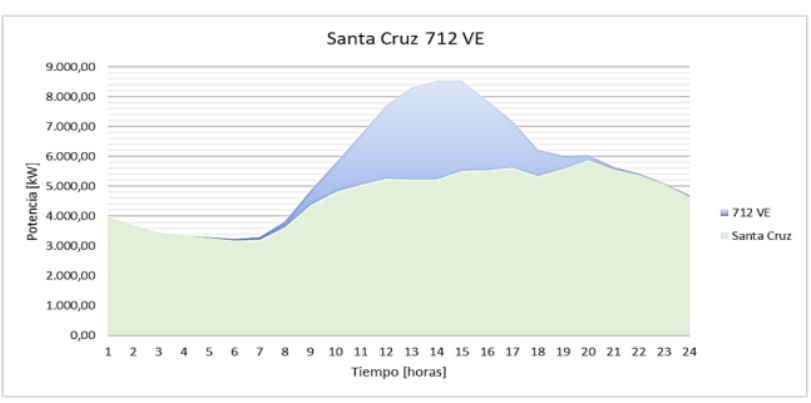

Figura 10: Demanda de potencia 712 vehículos eléctricos.

En el caso de Santa Cruz el mayor impacto lo provoca la inserción de autobuses eléctricos cuyo aporte a la demanda es de 2,49 MW, los $828 \mathrm{~kW}$ restantes de la demanda se deben a la inserción de SUV y automóviles eléctricos. La demanda crece a partir de las 8:00 y empieza a decaer alrededor de las 14:00 teniendo el pico máximo entre las 12:00 y 14:00 horas (Fig. 10).

La Figura 11 se refiere al caso de San Cristóbal y se muestra que los 100,74 kW no tienen mayor incidencia sobre la curva de demanda máxima registrada en 2018 y representa un incremento el $3 \%$ de la demanda máxima, la demanda crece a partir de las 8:00 h y empieza a decaer alrededor de las 15:00 h teniendo el pico máximo entre las 14:00 h y 16:00 h.

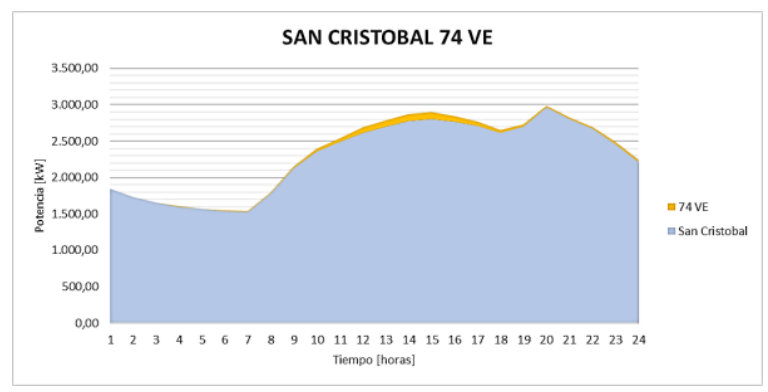

Figura 11: Demanda de potencia 74 vehículos eléctricos

\subsection{Estaciones de carga rápida}

\subsubsection{Santa Cruz}

De acuerdo con la Figura 9 y la Fig. 10 para Santa Cruz en ambos casos se observa que la mayor demanda de potencia se debe a 74 autobuses eléctricos, de estos el $14,3 \%$ (11 autobuses) cubrirá la ruta aeropuerto Isla Baltra-Baltra ferry terminal y el numero restante (63) de autobuses cubrirá las rutas Santa Cruz Ferry TerminalPuerto Ayora y Santa Cruz Ferry Terminal-Playa el Garrapatero. Se toma en cuenta los siguientes aspectos:

- Las estaciones de carga rápida atenderán las 24 horas del día.

- El tiempo de carga promedio será de 5 horas.

- La potencia de estos cargadores debe ser de $80 \mathrm{~kW}$.

- Las estaciones deben ser DC y modo 4

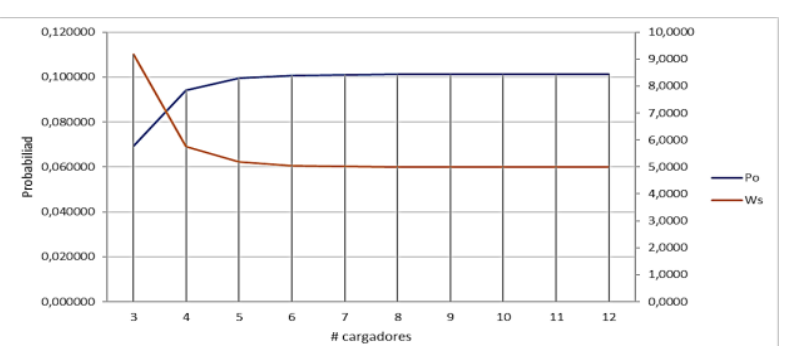

Figura 12: Método gráfico para 11 autobuses.

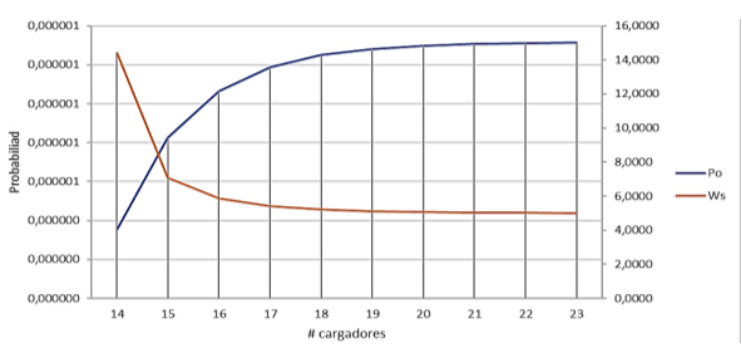

Figura 13: Método gráfico para 63 autobuses. 
En la Figura 12 y Figura 13 se muestra la determinación del número óptimo de cargadores para 11 y 63 autobuses eléctricos respectivamente.

\subsubsection{San Cristóbal}

De acuerdo con la Fig. 11 para San Cristóbal la mayor potencia de demanda se da debido a la inserción de 74 VE que cubrirán la ruta Puerto Baquerizo Moreno-Puerto Chino. Se toma en cuenta los siguientes aspectos:

- Las estaciones de carga rápida atenderán las 24 horas del día.

- El tiempo de carga promedio será de 1 hora.

- La potencia de los cargadores debe ser de una potencia de $24 \mathrm{~kW}$.

- La estación de carga debe ser tipo DC y modo 4.

En la Fig. 14 se muestra la determinación del número óptimo de cargadores para 74 vehículos eléctricos.

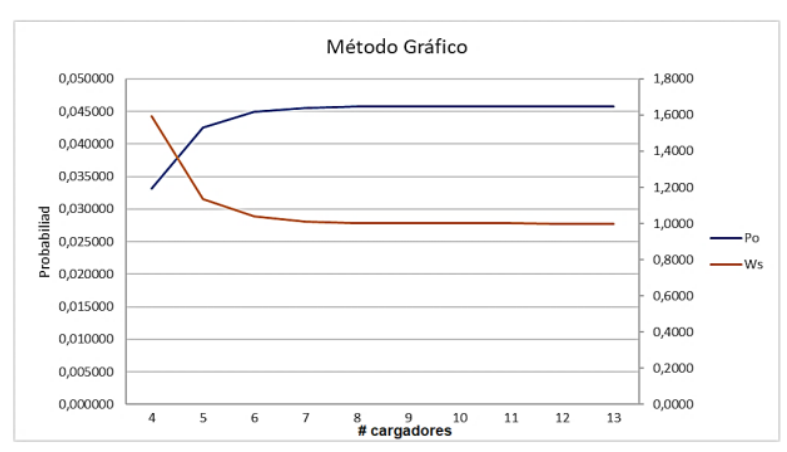

Figura 14: Método grafico para 74 vehículos eléctricos.

En la Tabla 3 se observa el número de cargadores y la ubicación de las estaciones de carga. La ubicación atiende a los lugares de mayor tránsito.

Tabla 3: Ubicación las estaciones de carga rápida en las Islas

\begin{tabular}{|c|c|c|c|}
\hline $\begin{array}{c}\text { Estación de } \\
\text { carga }\end{array}$ & $\begin{array}{c}\text { \# de } \\
\text { cargadores }\end{array}$ & $\begin{array}{c}\text { Potencia de } \\
\text { cargadores } \\
{[\mathbf{k W ]}}\end{array}$ & $\begin{array}{c}\text { Potencia } \\
\text { total [kW] }\end{array}$ \\
\hline $\begin{array}{c}\text { Aeropuerto } \\
\text { Baltra }\end{array}$ & 5 & 80 & 400 \\
\hline $\begin{array}{c}\text { Santa Cruz } \\
\text { Ferry Terminal }\end{array}$ & 9 & 80 & 720 \\
\hline Bellavista & 9 & 80 & 720 \\
\hline El progreso & 5 & 24 & 120 \\
\hline
\end{tabular}

\section{CONCLUSIONES Y RECOMENDACIONES}

El modelo planteado para la determinación de las curvas de carga para diferentes clases de VE, permite personalizar las consideraciones de acuerdo con los criterios presentes (incentivos tarifarios, incorporación de VE y tipos disponibles), de manera que las modelaciones y resultados finales puedan considerar las políticas vigentes o nuevos criterios de análisis.

La teoría de colas permite analizar el comportamiento de un servicio, en este caso de las estaciones en carga teniendo en cuenta: la cantidad de vehículos, comportamiento de llegada, salida, número de servidores, tiempo promedio de carga y tiempo de apertura de las estaciones de carga. En este caso se ha usado un modelo de cola estándar o simple.

Galápagos al ser una provincia relativamente pequeña, la ubicación de las estaciones de carga se realizó considerando las distancias desde los centros poblados hacia los lugares de mayor afluencia turística y tomando en cuenta el modelo de vehículo eléctrico con menor autonomía.

Los resultados del presente estudio pueden servir de guía para el desarrollo de una regulación que permita al ente de regulación establecer requisitos mínimos para estaciones de carga los cuales garanticen seguridad en la instalación y calidad en cuanto a términos eléctricos se refiera, ya que los planteamientos de esta propuesta tienen como base de soporte la norma internacional como ISO 17409 e IEC 61851-1.

A medida que la tecnología de vehículos eléctricos y estaciones de carga avanza se debería tomar en cuenta en futuros trabajo la incorporación de métodos de carga como lo son el método de carga inalámbrico y el método de intercambio de baterías, incluyendo un análisis técnico-financiero.

A futuro se podría incorporar en conjunto ambos modelos presentados, simulación de Montecarlo y teoría de colas, además de tomar en cuenta factores importantes como lo son la autonomía de los diferentes vehículos eléctricos y la degradación de la batería de estos.

\section{REFERENCIAS BIBLIOGRÁFICAS}

[1] Naciones Unidas, "Sutainable development goals," [En línea]. Available: https://www.un.org/sustainabledevelopment/climat e-change/. [Último acceso: 28 Diciembre 2020].

[2] International Energy Agency, "Electric vehicles," [En línea]. Available: https://www.iea.org/reports/electric-vehicles. [Último acceso: 28 Diciembre 2020].

[3] International Economic Development Council, "Analysis of the Electric Vehicle Industry," Washington DC, 2013.

[4] Electric vehicle conductive charging system. Part 1: General Requirements, IEC 61851-1, 2017.

[5] Electrically propelled road vehicles - Connection to an external electric power supply - Safety requirements, ISO 17409:2015, 2015.

[6] Electric vehicle conductive charging system - Part 23: DC electric vehicle charging stations, IEC 61851-23:2014, 2014.

[7] Electric Vehicle Conductive for DC charging system, AIS-138 (Part 2), India, 2018. 
[8] Electrically propelled road vehicles-Safety Specifications-Part 3: Electrical safety, ISO 6469-3, 2018.

[9] National Electrical Code, 2014 ed, NFPA, USA, 2014, pp. 1046-1043.

[10] Norma ecuatoriana de construcccion-Instalaciones electromecánicas, ed. 2013, MIDUVI, Ecuador, 2013, pp.14-71.

[11] IEEE guide for safety in AC substation Grounding,Std. IEEE 80-2000, 2000 .

[12] J. Bablo, "Electric Vehicle Infrastructure Standardization", World Electric Vehicle Journal, 8(2), 576-586, DOI:10.3390/wevj8020576.

[13] Guidance for implementation of electric vehicle charging infrastructure, Local Government of London, 1st ed., London, 2010, pp. 8-14.

[14] Electric Vehicle (EV) Charging Infraestructure, 1st ed, Queensland Government, Queensland, 2018, pp. 2-11.

[15] MC. Falvo, D. Sbordone, IS. Bayram, M. Devetsikiotis, "Estaciones y modos de carga EV: estándares internacionales", Simposio internacional 2014 sobre electrónica de potencia, accionamientos eléctricos, automatización y movimiento, DOI: 10.1109 / speedam.2014.6872107.

[16]Electric Vehicle Charging Stations-Technical Installation Guide, 2nd ed., Hydro Québec, Quebec, 2015, pp.10-21

[17] Pod Point, "Electric Vehicle Guides", noviembre de 2019. [En línea]. Disponible en: https://podpoint.com/guides/vehicles? [Accedido: diciembre-2019].

[18]C. Azofeifa, “Aplicación de la Simulación MonteCarlo", Tecnología en Marcha, 1era ed., vol. 1, Costa Rica.

[19] J. Heizer, B. Render, "Modelo de colas (líneas de espera)", en Direccion de la produccion y de operaciones, 8va ed., vol. 1, ES: Perason Prentice Hall, 2007, pp. 401-421.

[20]E. Calle, "Determinación del factor de coincidencia y curva de carga de vehículos eléctricos en base a mediciones, comparación con el caso de Galápagos”, Agencia de Regulación y Control de Electricidad, Quito, Ecuador, 2019.

[21] A. Ganti, "Correlation Coefficient", 14 de agosto de 2019. [En línea]. Disponible en: https://www.investopedia.com/terms/c/correlationc oefficient.asp. [Accedido: 2-oct-2019].

[22] Estadísticas del Sector del transporte 2018, Evolución Histórica del Anuario de Estadísticas de Transporte, Ed. 2018, Instituto Nacional de
Estadísticas y Censos, Quito, Ecuador, 2019.

[23] Catastro 2018 de uso de la energía eléctrica, Rendición de cuentas 2018, Empresa Eléctrica Galápagos, Galápagos, Ecuador, 2019.

[24] J. Heizer, B. Render, "Modelo de colas (líneas de espera)", en Direccion de la produccion y de operaciones, 8va ed., vol. 1, ES: Perason Prentice Hall, 2007.

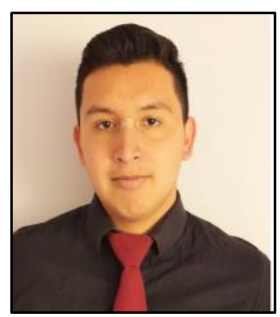

Luis Andrés Mera. - Realizó sus estudios secundarios en el Colegio Técnico Salesiano Don Bosco". Obtuvo su título de Bachiller Técnico en Instalaciones, Equipos y Máquinas Eléctricas en julio del 2013. Realizó sus estudios superiores en la Escuela Politécnica Nacional en la carrera de Ingeniería Eléctrica. Sus áreas de interés son: Energías Renovables no Convencionales, Distribución de Energía Eléctrica y Transmisión de Energía Eléctrica.

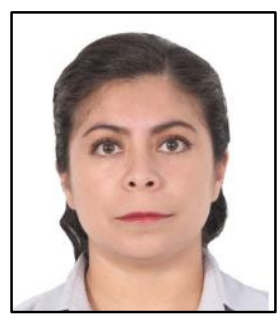

Patricia Elizabeth Otero. - Es Ingeniera en Electrónica y Control de la Escuela Politécnica Nacional, tiene una maestría en Ingeniería Eléctrica y una en Administración de Empresas, de la misma institución. Ha desempeñado cargos como: Gerente del Proyecto de Electrificación Rural para Zonas Aisladas del Ecuador, Jefe de Transacciones Comerciales de Energía para la Empresa Pública Metropolitana de Agua Potable y Saneamiento, Asesora en Energía Renovable y Eficiencia Energética para la Empresa Eléctrica Quito. Actualmente es profesora del Departamento de Energía Eléctrica de la Escuela Politécnica Nacional.

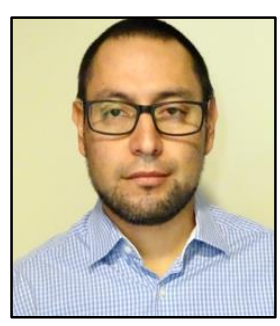

Víctor E. Calle García. Ingeniero Eléctrico, de la Universidad Politécnica Salesiana, tiene un diplomado en Economía de la Regulación de la Universidad de San Andrés, Argentina; es especialista en Administración de Empresas. Ha desempeñado cargos como: Jefe de Control de Pérdidas en la Corporación Nacional de Electricidad, Asesor Eléctrico en la Secretaría Nacional de Planificación y Desarrollo, especialista de planificación de los sistemas de distribución en el Consejo Nacional de Electricidad. Actualmente labora en la Agencia de Regulación y Control de Electricidad, como especialista en planificación de distribución y movilidad eléctrica. 